\title{
IMPROVing PACKet Delivery RATio With ENHANCED CONFIDENTIALITY IN MANET
}

\author{
S.VASUDEVI ${ }^{1}$ AND D.MARYPONRANI ${ }^{2}$ \\ ${ }^{1}$ Final year PG Student, ${ }^{2}$ Assistant Professor \\ Department of Computer Science Engineering, \\ Dr.Sivanthi Aditanar College of Engineering, Tiruchendur, India
}

\begin{abstract}
In Mobile Ad Hoc Network (MANET), the collection of mobile nodes gets communicated without the need of any customary infrastructure. In MANET, repeated topology changes and intermittent link breakage causes the failure of existing path. This leads to rediscovery of new route by broadcasting RREQ packet. The number of RREQ packet in the network gets added due to the increased amount of link failures. This result in increased routing overhead which degrades the packet delivery ratio in MANET. While designing routing protocols for MANET, it is indispensable to reduce the overhead in route discovery. In our previous work [17], routing protocol based on neighbour details and probabilistic knowledge is utilized, additionally the symmetric cipher AES is used for securing the data packet. Through this protocol, packet delivery ratio gets increased and confidentiality is ensured. But there is a problem in secure key exchange among the source and destination while using AES. To resolve that problem, hybrid cryptographic system i.e., combination of AES and RSA is proposed in this paper. By using this hybrid cryptographic scheme and the routing protocol based on probability and neighbour knowledge, enhanced secure packet delivery is ensured in MANET.
\end{abstract}

\section{KEYWORDS}

MANET, routing protocols, routing overhead, Packet delivery ratio, cryptography algorithms.

\section{INTRODUCTION}

In Mobile Ad hoc networks, the nodes are considered to be mobile and the communication among those nodes are done through dynamic routes. Here the route initially discovered gets change from time to time because of the nodes mobility. This redundant route discovery causes the heavy routing overhead and result in huge number of packet loss. Consequently it affects the delivery rate of packets. So there is a great challenge in designing the routing protocol that suits host mobility and link failure in MANET

The routing protocol in MANET are divided into three categories such as Proactive routing, Reactive routing and Hybrid routing protocol. In Proactive routing, every node computes the path to every other node in the MANET and stored it in its own routing table. This routing table is periodically updated whenever changes happened in the route among the nodes. In Reactive routing, path between the nodes are computed only when the demand for the route is needed. This routing protocol is also termed as On-demand routing protocol. The third category Hybrid routing combines both proactive and Reactive routing principles.

AODV and DSR are some of the conventional On-demand routing protocol in MANET. Both of them[2] [4] uses pure flooding for route discovery. In Pure flooding, every node that receives the 
route request packet can rebroadcast it to its neighbours if it is not the intended destination. This results in duplication of request packet in some nodes. This kind of redundant retransmission leads to contention, collision and redundancy and leads to the storm problem.

To Reduce this storm problem, several methods [1] like area-based methods ,probability-based methods, flooding, neighbor-knowledge method are introduced. Probabilistic Scheme, Counter based Scheme and Distance based Schemes are used to shrink the effect of storm problem [1]. In Probabilistic Scheme, RREQ is forwarded depends on the determined probability value. In Counter based Scheme, forwarding decisions are taken by Expected Additional Coverage (EAC). In Distance based scheme, node's distances are used to decide the packet retransmission.

On demand Tree based Routing and Scalable Broadcasting Algorithm are some techniques which helps to reduce redundant retransmission. By means of using Probabilistic routing, packet loss due to routing overhead is somewhat reduced, but there is a problem in fixed probabilistic value. By combining both neighbor knowledge and probabilistic method[10], problems in the DPR[9] is solved and also Packet delivery rate gets increased. Therefore in our work NCPR[10] is selected as a routing protocol.

By means of NCPR, defects in pure flooding are neglected, this also leads to increased Packet delivery ratio. Additional protection in Packet delivery is obtained by using effective security mechanisms like hybrid cryptographic techniques for encrypting and decrypting the data packets. Here the combination of AES (Advanced Encryption Standard)[12] and RSA (Rivest-ShamirAdleman) [15] are used for ensuring secure delivery of the data packets. By this, confidentiality is also maintained.

\section{RELATED WORKS}

The reason for using Ad hoc network widely is its quick deployment nature and its less dependency on external infrastructure. But routing is the serious issue because of the nodes frequent movement. Some of the conventional routing protocols AODV and DSR are discussed in the reference papers [1] and [2]. AODV proposed by C. Perkins, E. Belding-Royer, and S. Das [1] is intended for use in an ad hoc network and it provides quick adaptation to dynamic link conditions. This protocol solves the count to infinity problem that occurs because of routing loops in MANET. This protocol fails to solve the routing overhead and hello packet overhead and thus affects packet delivery ratio in MANET.

The other conventional routing protocol DSR proposed by D. Johnson, Y. Hu and D. Maltz [2] supports efficient routing in MANET. In the header of the RREQ packet, source route is maintained in DSR. This leads to higher packet size and sometimes the performance of the network is affected because of the presence of stale cache.

Broadcast Storm problem in MANET is described by S.Y. Ni, Y.C. Tseng, Y.S. Chen, and J.P. Sheu in reference paper [4]. S.Y. Ni, et.al.., propose several schemes to reduce redundant rebroadcasts and to vary the timing of rebroadcasts to solve the storm problem. Probabilistic scheme, Distance-based scheme, Counter based scheme and Cluster based schemes are proposed in[4]. In Probabilistic Scheme[5] rebroadcast is done based on the computed probability value. If determined probability value is equal to 1 , it is similar to pure flooding. In Counter-Based Scheme[4] and [5], the counter threshold value is used to decide rebroadcast of RREQ. In that paper, to control the unnecessary rebroadcast of RREQ Expected Additional Coverage(EAC) is used. Retransmission is prevented in case of very low EAC value. 
Scalable Broadcasting Algorithm (SBA) proposed by W. Peng and X. Lu [8] aims to reduce the broadcast redundancy in MANET. By using the redundant broadcast and local topology information, unnecessary broadcasts are avoided. Thus network energy and bandwidth gets less consumed. The main idea of SBA is "the node need not rebroadcast the received RREQ if all of its neighbours have been covered by previous RREQ".

OTRP proposed by H. AlAamri,M.Abolhasan, and T.Wysocki uses Tree-based Optimized Flooding (TOF) algorithm to improve the scalability even though previous knowledge to the destination is not available in ad hoc network.

The basic probabilistic route discovery method FPR discussed in [9] use the fixed probability value without considering network density. In Fixed Probabilistic Routing, source can broadcast RREQ with probability "P=1" i.e.,( simple flooding). Any neighbour node that receives that RREQ, rebroadcast it to its neighbours with probability value less than 1 . The probability value used in FPR is same for all nodes and it does not varied based on the local topology features. It result in unfair distribution of probability and to resolve this problem, forwarding probability needs to be determined based on random distribution of mobile nodes and regions of varying degrees of node density.

Dynamic Probabilistic route discovery (DPR) proposed by J.D. Abdulai, M. Ould-Khaoua, L.M. Mackenzie, and A. Mohammed [9] uses dynamic forwarding probability values. These values are found by considering local density of forwarding node and set of its covered neighbours. By finding density of region in network using local neighbourhood information of that region, local density is estimated.

Sung Xin ,Jing Xia,En Bo Wang and Dan Keun proposed NCPR [10] which gives good delivery ratio compared to DPR. Performance evaluation of symmetric encryption algorithms in MANET like AES,DES,etc.., are computed by Md.MohirHossain and M.A.Matin in [12]. Xin Zhou and Tang discuss about the RSA implementation and benifits of using those public key encryption algorithm in [15]. In our previous work [17], NCPR with AES is used to render secure packet delivery ratio. But there is a problem in secure key exchange of AES, since it is the private key encryption algorithm. To resolve that problem,i.e., for ensuring secure AES key exchange, a public key encryption algorithm RSA is used in our paper.

\section{PROTOCOL DESCRIPTION}

In this section, the protocol used for reducing the overhead during routing and security mechanisms added to the data packet are discussed. Neighbor Coverage based Probabilistic Routing protocol (NCPR) from reference paper [10] is used for effective routing and improved packet delivery ratio. NCPR protocol can solves the redundant rebroadcast by forwarding the packet with the rebroadcast probability that is computed from additional coverage ratio and connectivity factor. Also the combination of symmetric and asymmetric cryptographic mechanisms are added to ensure secure packet delivery.

\subsection{Uncovered Node Computation}

When the RREQ packet is broadcasted from source node, the neighbour nodes that receives the RREQ can perform the uncovered neighbour computation to calculate the set of its neighbours that doesn't have the RREQ flooded from the source.

Initial Uncovered neighbour computation is done using the below formula :

$$
\mathrm{UCN}\left(n_{x}\right)=\mathrm{N}\left(n_{x}\right)-\left[\mathrm{N}\left(n_{x}\right) \cap \mathrm{N}(\text { source })\right]-\{\text { source }\}
$$


Where $\operatorname{UCN}\left(n_{x}\right)$ denotes uncovered neighbours of node. $n_{x}$ is one of the neighbour node of source node. 'source' denotes the source node which broadcast the RREQ packet. $\mathrm{N}\left(n_{x}\right)$ represents the neighbours of node $n_{x}$ and $\mathrm{N}$ (source) denotes the neighbours of source node.

This computation takes the difference of neighbours of node $n_{x}$ and common neighbours of both $n_{x}$ and source.

\subsection{Rebroadcast Delay and Rebroadcast Probability Calculation}

After the computation of initial UCN, RREQ rebroadcast is delayed for some time interval. This delay value is called Rebroadcast delay and using it rebroadcast order is determined .

According to [10], Rebroadcast delay is computed using below formula :

$$
\operatorname{RBD}\left(n_{x}\right)=\text { MaxDelay } \times\left(1-\frac{\mid \mathrm{N}(\text { source }) \cap \mathrm{N}\left(n_{x}\right) \mid}{\mid \mathrm{N}(\text { source }) \mid}\right)
$$

Where $\operatorname{RBD}\left(n_{x}\right)$ denotes rebroadcast delay of node $n_{x}$. A small constant delay is used as MaxDelay.

The node can set its timer based on that rebroadcast delay value. By using this delay time, efficient transmission order is determined. If any redundant RREQ packet is received in from its neighbour $n_{y}$ during this delay time, then the node $n_{x}$ can adjust its neighbour list in UCN as,

$$
\mathrm{UCN}\left(n_{x}\right)=\mathrm{UCN}\left(n_{x}\right)-\left[\mathrm{UCN}\left(n_{x}\right) \cap \mathrm{N}\left(n_{y}\right)\right]
$$

The duplicate RREQ packet received from the node $n_{y}$ is discarded after this adjustment. When this delay timer gets expired, the node can gain its final UCN set. Rebroadcast probability is determined by taking the product of additional coverage ratio and connectivity factor. Additional coverage ratio is the ratio of uncovered neighbours of node $n_{x}$ to neighbours of the node $n_{x}$. It is computed using below formula :

$$
\operatorname{ACR}\left(n_{x}\right)=\frac{\left|\mathrm{UCN}\left(n_{x}\right)\right|}{\left|\mathrm{N}\left(n_{x}\right)\right|}
$$

Connectivity factor (CF) is obtained by dividing connectivity metric $5.1774 \log (\mathrm{n})$ by total number of neighbours of node $n_{x}$, where $\mathrm{n}$ is the number of nodes in the MANET. The formula for Connectivity factor is given as below :

$$
\mathrm{CF}\left(\left(n_{x}\right)=\frac{5.1774 \log (\mathrm{n})}{\left|\mathrm{N}\left(n_{x}\right)\right|}\right.
$$

\subsection{Adding Security to Data Packet}

In this paper, the data packets are secured using symmetric key algorithm to ensure confidentiality. Here AES is used for encryption purposes. To solve the problem in symmetric key distribution, an asymmetric algorithm RSA is used for secure transfer of AES key. Thus the hybrid scheme is utilized for better results in secure delivery of packets.

AES stands for Advanced Encryption standard and is based on Rijndael cipher. It is one of the symmetric cipher algorithm. It stores the compressed form of encrypted data resulted in small size database. It provides faster encryption and decryption as well as confidentiality. But the 
problem in symmetric cryptography is lack of security during key exchange between the source and destination, since single key is used for both encryption and decryption. With the aim of solve that symmetric key exchange problem, asymmetric key encryption technique named RSA is utilized here.

RSA stands for Rivest Shamir and Adleman and it uses two keys (i.e.) public and private key for encryption and decryption. Every source node can have its own public key and private key. The source node encrypts the data using destination node's public key and then the destination node that receives the data can decrypt it using his own private key. By having these two keys, it overcomes the secure key distribution problem occured in symmetric crypto system. But RSA consumes more encryption time and it's memory usage is also high. RSA is not preferred for data encryption. AES does the data encryption in a reasonable time as compared to RSA, the only problem is the failure in exchanging the key securely. So in this paper, RSA is used for encrypting the AES key at the sender side and decrypting that at the receiver side. Through this hybrid technique, secure packet delivery is ensured.

\subsection{Flow of Algorithm}

The route between the source 'Src' and the destination 'Dest' is computed using NCPR [10] protocol. Then the data packet to be sent to the destination is encrypted using AES. Then the symmetric key used by AES is encrypted by RSA. Once the encrypted AES key is received at the destination, it can be decrypted using RSA thus obtains the Key to decrypt the data packet. Then by using that key, data is decrypted by AES scheme. The flow of the algorithm are given below :

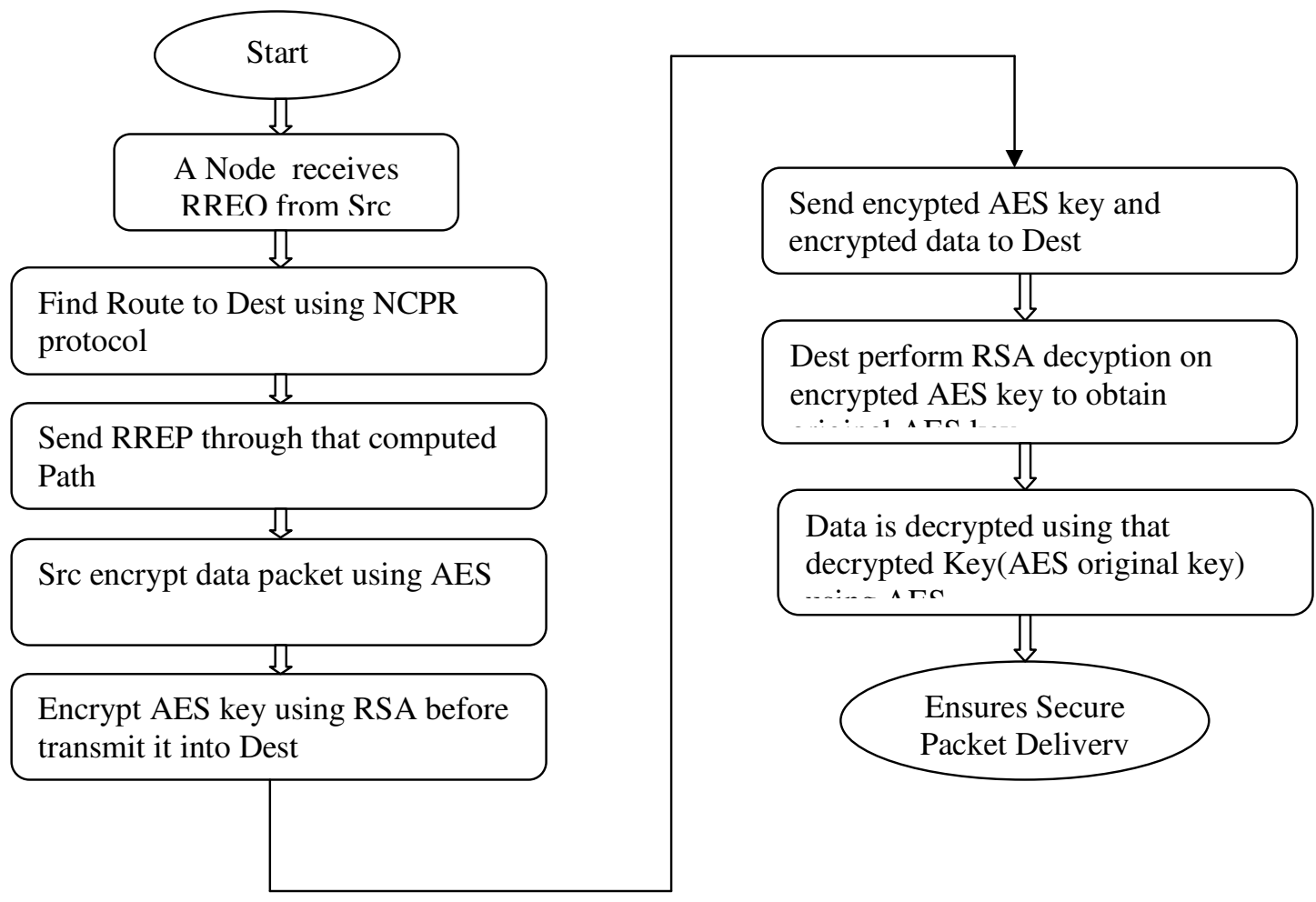

Figure 1. Flow of Algorithm 


\subsection{Implementation of Protocol}

According to [10], the source code of AODV in NS-2 is modified to implement NCPR. By means of using this protocol, hello packet overhead and overhead occurred due to the neighbor list in RREQ are reduced.

The hello packet overhead is reduced by restricting the use of periodical hello mechanism. Instead of hello packets, RREQ and RERR control packets are used. The mechanism specified in [11] helps for hello packet overhead reduction. According to that paper [11], the node can send hello packet only if the elapsed time of RREQ is greater than hello interval value.

In RREQ packet, the neighbor list overhead is reduced by monitoring the changes of the node's neighbor table. RREQ header of the existing AODV is modified by inserting a fixed field that represents the size of neighbor list in RREQ and dynamic neighbor list.

As the implementation of NCPR gets succeeded, the next step is to apply the AES algorithm to encrypt the data Packet. Then the AES key is encrypted using RSA. At receiver side, AES key is decrypted by RSA and using that key encrypted data packet is decrypted using AES.

\section{NeTWORK ANALYSIS AND Simulation ReSUltS}

For simulate this protocol, NS-2 (v2.30) is used. The topology size is set as $1100 \mathrm{~m} \times 1100 \mathrm{~m}$. The number of nodes may be set as 50,100 or 150 . The transmission range of each node is set as $250 \mathrm{~m}$. The constant Bit Rate (CBR) traffic is used. The number of CBR connections may be $5,10,12$ or 14 . The size of packet is 512 bytes and packet rate is 4 packets/sec. The minimum speed is $1 \mathrm{~m} / \mathrm{sec}$. The maximum speed is $5 \mathrm{~m} / \mathrm{sec}$. The simulation time is set as 150 seconds.

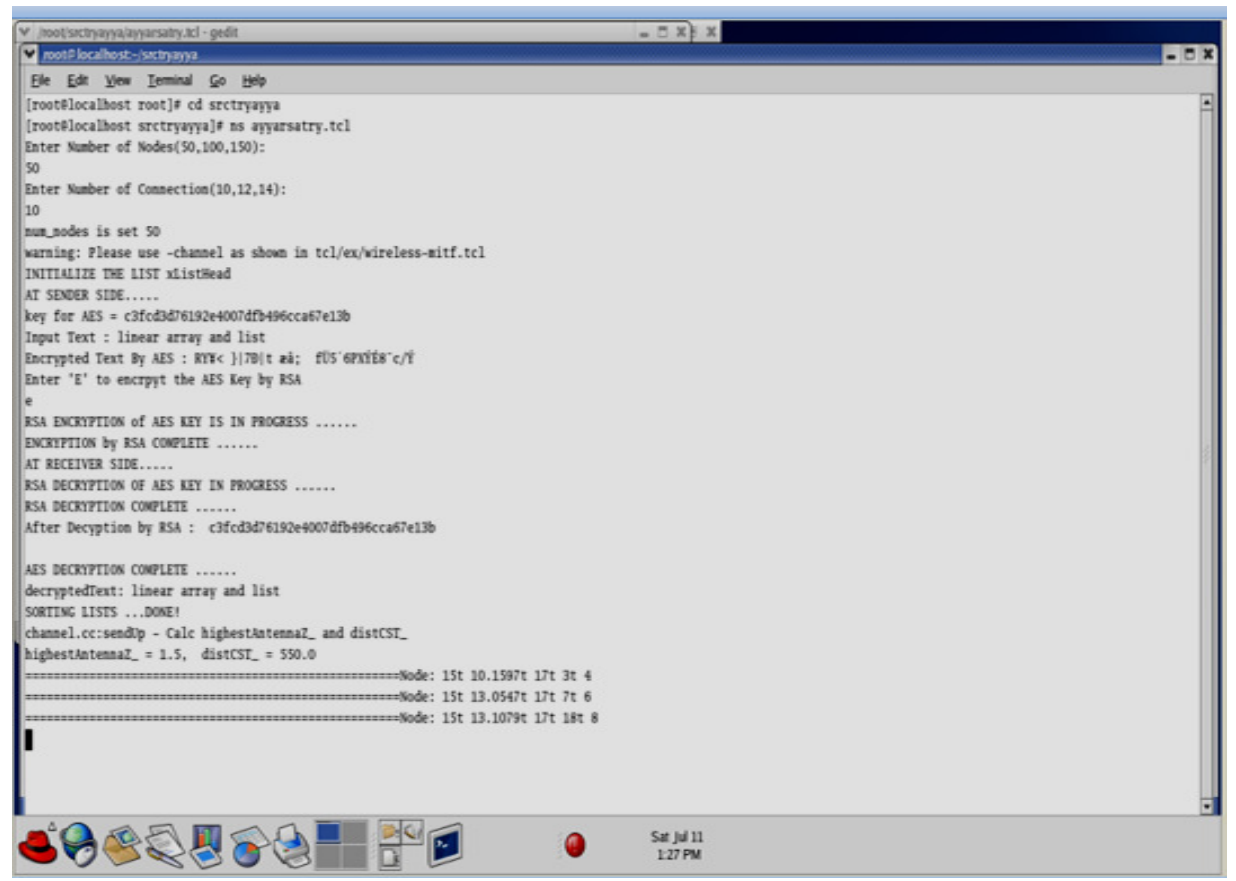

Figure 2. Nodes and Connection details and Hybrid encryption and decryption 
Initially the number of nodes and CBR connections needed are to be selected. Once these details are gets selected, MANET with selected number of nodes and CBR connections are configured and route discovery among every source and destination are done via NCPR [10] protocol. Then the random input string and key is gets generated. Using that key value, that random input text is encrypted at sender side using AES. After that, the key used for AES encryption is encrypted using RSA for securing confidentiality of AES Key while transmission. At receiver side, that key (encrypted by RSA) is decrypted using RSA decryption, thus obtains the key for decrypting the data(input text). Using that decrypted key, data is decrypted by AES decryption. The changes happened in the routing table are also displayed. These are depicted in Figure.2

In above window(figure.2), number of nodes are selected as 50 and number of CBR connections are selected as 10 . This denotes 10 different source and destination gets communicated within the simulation time among 50 nodes. The communication among each source and destination are done via NCPR routing protocol[10].

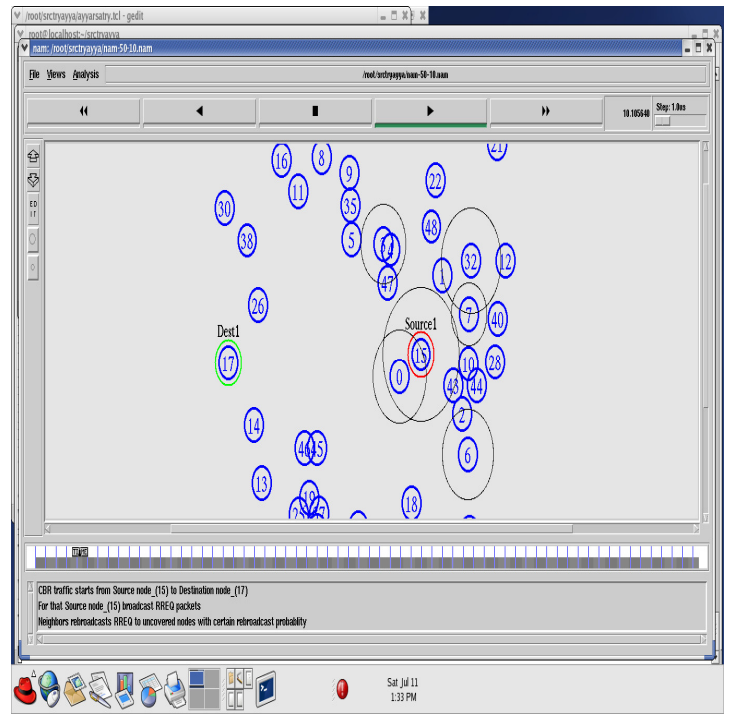

Figure 3.a

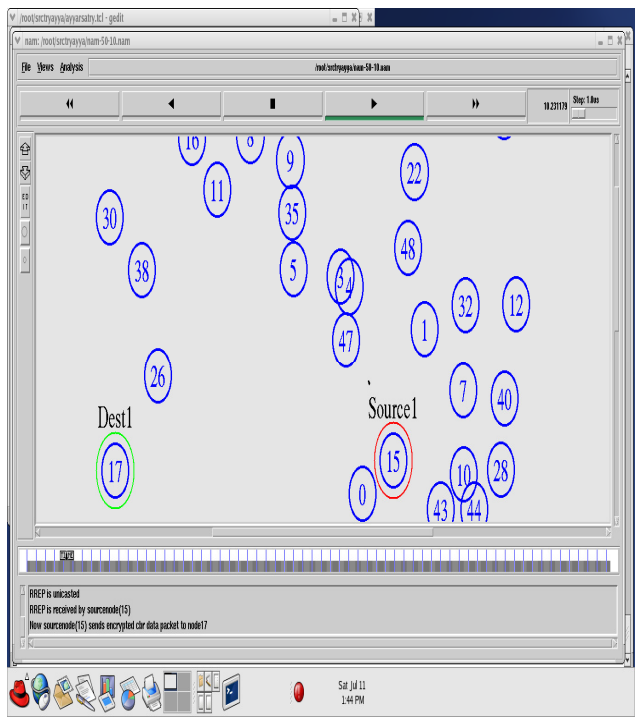

Figure 3.b

Figure 3.(a)Route discovery (RREQ packet rebroadcast to Uncovered node with Rebroadcast Probability), (b) Source 15 send encrypted data into destination node 17

Then CBR connections are started between source 15 and destination 17. So node 15 starts broadcasted RREQ to reach node 17. The nodes that receives the RREQ can rebroadcast it to uncovered neighbors with computed rebroadcast probability value as shown in figure 3.a.The broadcasted RREQ packet reaches the destination node 17 and RREP starts unicasted. After the RREP reach the source, the source node 15 sends the encrpted data packet to destination node 17 through the path from where RREP comes as shown in figure 3.b. As a denotion of successful delivery of data packets, CBR packet gets received by the destination through that path. In case of any packet drop due to the node movement, Route rediscovery occurred by following the same above route discovery process. 
The Uncovered Neighbors(UCN) for each neighbor nodes of node 15 are computed and it gets stored in one trace file. Then based on this UCN details, Additional Coverage Ratio(ACR) and Connectivity Factor(CF) are computed. From that, Rebroadcast delay and Probability gets computed by taking the product of ACR and CF as shown below.

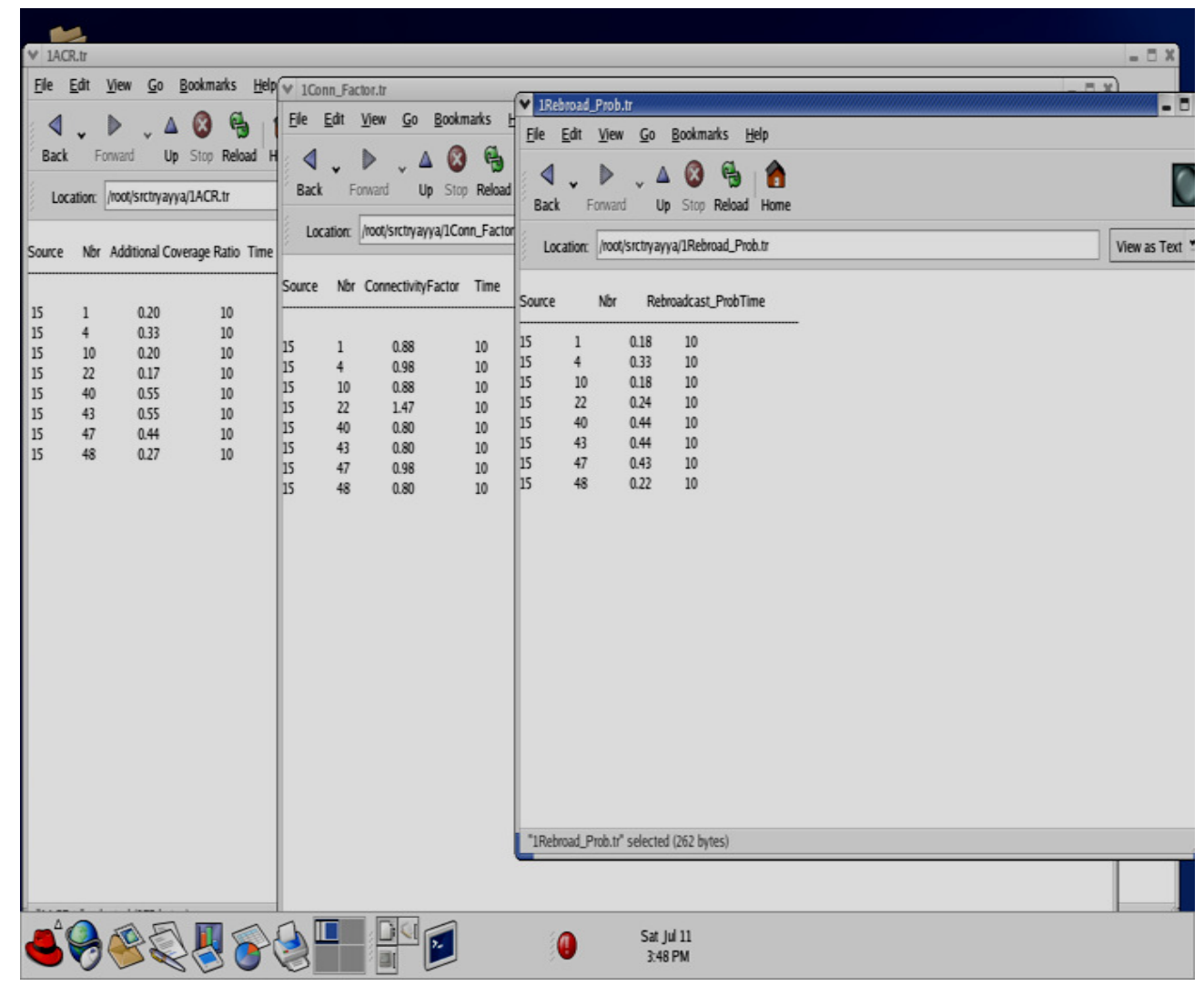

Figure 3. ACR,CF and Rebroadcast Probability for neighbors of 15 at time 10.

The implemented secure routing protocol i.e., Hybrid secure-NCPR(It uses both RSA and AES with exixting NCPR) is evaluated by means of checking the packet delivery ratio obtained by varying number of CBR connections.

Packet Delivery Ratio shortly termed as PDR is the ratio of the number of data packets successfully arrived in the CBR destinations to the number of data packets generated by the CBR sources.

The Packet Delivery Ratio obtained using 50 nodes and 10 connections are computed. Then by varying the connections into 5,12 and 14, corresponding PDR value is calculated. The packet delivery ratio obtained as a result of varying number of CBR connections are given in figure 4 .

Also the PDR value of implemented Hybrid secure-NCPR protocol is compared with PDR values of existing protocols like DPR and AODV. As a result of comparison, It is concluded that Hybrid secure-NCPR gives better results in Packet delivery than DPR and AODV. 


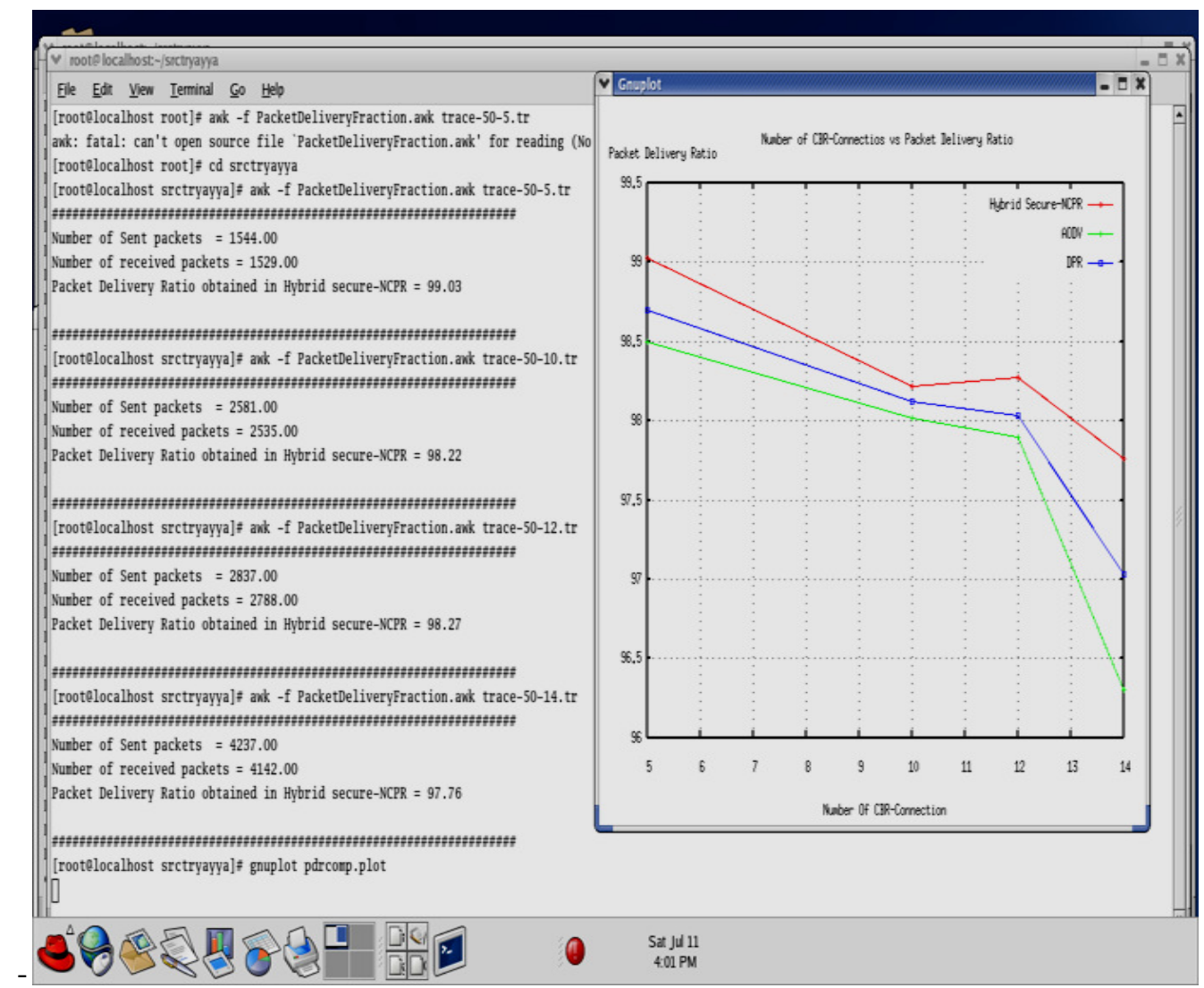

Figure 4. PDR for proposed method by varying CBR connections and Comparison PDR Graph among proposed protocol, AODV and DPR

\section{CONCLUSIONS}

In this paper, the routing protocol that reduces overhead problem occurred during route discovery in MANET for improving the packet delivery ratio is discussed. By using this protocol, broadcast storm problem that causes the serious contention, collision and redundant rebroadcast in the network is resolved, thus reduces the routing overhead and ensures high packet delivery. Also the combination of symmetric and asymmetric cipher algorithm (AES and RSA) is utilized to ensure secure packet delivery. Using AES algorithm, the data packets flows from source to destination are encrypted, thus confidentiality is guaranteed. And AES key is securely transmitted via RSA algorithm. Thus enhanced secure Packet delivery is achieved in MANET. QOS consideration of this protocol will be left for future enhancement.

\section{REFERENCES}

[1] S. Das, E. Belding-Royer, C. Perkins, “AdHoc On-Demand Distance Vector(Proactive routing) Routing", RFC 3561, 2003.

[2] Y. Hu, D. Maltz \& D. Johnson, "The Dynamic Source Routing Protocol for Mobile Ad Hoc Networks for IPversion 4”, IETF RFC 4728, 2007, vol. 15, pp. 153-181,2009.

[3] T. Wysocki,, M. Abolhasan and H. AlAamri, “On Optimising Route Discovery in Absence of Previous Route Information in MANET(OTRP)", VTC in IEEE, 2009. 
International Journal on Computational Science \& Applications (IJCSA) Vol.5, No.5,October 2015

[4] Y. Ni, Sheu.J.P, Chen, "The Broadcast Storm Problem in a Mobile AdHoc Network", IEEE/ACM MobiCom,1999.

[5] Mackenzie, A. Mohammed, Perkin, and J.D.Abdulai, "Probabilistic Counter-Based Route Discovery for Mobile Ad Hoc Networks", Proceedings. Int'l Conf. Wireless Comm. And Mobile Computing: Connecting the World Wirelessly (IWCMC'09), pp. 1335-1339, 2009.

[6] B. Williams and T. Cam , "Comparison of Broadcasting Techniques for Mobile Ad hoc Networks", Proceedings. ACM MobiHoc, pp. 194-205,2002.

[7] Mackenzie ,J.D. Abdulai, and M. Ould-Khaoua, "Improving Probabilistic Route Discovery in Mobile Ad Hoc Networks", Proceedings IEEE Conf. Local Computer Networks, pp. 739-746, (2007).

[8] X. Lu and Peng.w, "On the Reduction of Broadcast Redundancy in Mobile Ad Hoc Networks", Proceedings, ACM MobiHoc, pp. 129-130, 2000.

[9] L.M. Mackenzie, J.D. Abdulai, M. Ould-Khaoua, and A. Mohammed, "Neighbour Coverage: A Dynamic Probabilistic Route Discovery for Mobile Ad Hoc Networks", Proceedings. Int'1 Symp.Performance Evaluation of Computer and Telecomm. Systems (SPECTS '08), pp. 165$17,2008$.

[10] Sung Xin , Jing Xia, En Bo Wang and Dan Keun, “A Neighbor Coverage-Based Probabilistic Rebroadcast for Reducing Routing Overhead in Mobile Ad Hoc Networks”, proceedings $\operatorname{IEEE}(2013)$

[11] Sung, Wang, X.M .Zhang., “An Estimated Distance Based Routing Protocol for Mobile Adhoc Networks”, proceedings. IEEE Trans. Vehicular Technology, no. 7, pp. 3473-3484, Sept.2011.

[12] Md.MohirHossain and M.A.Matin, "Performance Evaluation of Symmetric Encryption Algorithm in MANET and LAN", Proceedings.IEEE Technical postgraduates International conferernce, 2000.

[13] Badache.N, Khelladi.L, D.Djenouri, “A survey of security issues in mobile ad hoc and sensor networks", Proceedings. IEEE Communications Surveys and Tutorials Journal, pp 2-29, (December 2005).

[14] W., Agrawal, H.,Li. Deng, "Routing security in wireless ad hoc networks", Proceedings IEEE Communications Magazine 40.pp . 70-75, (October 2002).

[15] Xin Zhou and Xiaofei Tang, "Research and Implementation of RSA Algorithm for Encryption and Decryption", the 6th International Forum on Strategic Technology, pp. 1118 - 1121, 2011.

[16] Gurpreet Singh, Supriya, "A Study of Encryption Algorithms (RSA, DES, 3DES and AES) for Information Security”, International Journal of Computer Applications (0975 - 8887) Volume 67No.19, April 2013

[17] S.Vasudevi, Ms.D.MaryPonrani, "Protocol for Improving Packet Delivery Ratio in MANET" in International Journal of Advance Research In Science And Engineering, Vol. No.4, Special Issue (02), March 2015,ISSN-2319-8354(E), Pg. No: 96-104 